\title{
Better Islet Function and Cardiovascular Autonomic Function in Type 2 Diabetic Patients With Pure Small Fibre Neuropathy Than With Mixed Neuropathy
}

\section{Chenxi Li}

Nanjing Drum Tower Hospital: Nanjing University Medical School Affiliated Nanjing Drum Tower Hospital

\section{Weimin Wang}

Nanjing Drum Tower Hospital: Nanjing University Medical School Affiliated Nanjing Drum Tower Hospital

\section{Wenyu Ni}

Nanjing Drum Tower Hospital: Nanjing University Medical School Affiliated Nanjing Drum Tower Hospital

\section{Yu Jin}

Nanjing Drum Tower Hospital: Nanjing University Medical School Affiliated Nanjing Drum Tower Hospital

\section{Simin Guo}

Nanjing Drum Tower Hospital: Nanjing University Medical School Affiliated Nanjing Drum Tower Hospital

\section{Jiewen Jin}

Sun Yat-sen University First Affiliated Hospital

\section{Chuhui Chen}

Fujian Provincial Hospital

\section{Wei Chen}

Nanjing Drum Tower Hospital: Nanjing University Medical School Affiliated Nanjing Drum Tower Hospital

\section{Yan Bi ( $\nabla$ biyan@nju.edu.cn )}

Drum Tower Hospital affiliated to Nanjing University Medical School https://orcid.org/0000-00033914-7854

\section{Dalong Zhu}

Nanjing Drum Tower Hospital: Nanjing University Medical School Affiliated Nanjing Drum Tower Hospital 
Original investigation

Keywords: Small fibre neuropathy, diabetic polyneuropathy, cardiovascular autonomic neuropathy, type 2 diabetes mellitus, skin biopsy

Posted Date: January 15th, 2021

DOI: https://doi.org/10.21203/rs.3.rs-144448/v1

License: (c) (i) This work is licensed under a Creative Commons Attribution 4.0 International License.

Read Full License 
Better islet function and cardiovascular autonomic function in type 2 diabetic patients with pure small fibre neuropathy than with mixed neuropathy

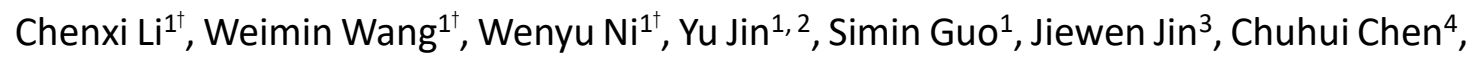
Wei Chen ${ }^{1}$, Yan Bi ${ }^{1 *}$, Dalong $\mathrm{Zhu}^{1^{*}}$

${ }^{\dagger}$ Chenxi Li, Weimin Wang, and Wenyu Ni equally contributed to this work.

*Correspondence: biyan@nju.edu.cn; zhudalong@nju.edu.cn

Yan Bi and Dalong Zhu are co-corresponding authors.

\begin{abstract}
Background

The clinical characteristics and outcomes of small fibre neuropathy (SFN) in Chinese patients with type 2 diabetes have not been thoroughly described, and we investigated metabolic and neurological indexes and the prognosis of type 2 diabetic patients based on skin biopsy.
\end{abstract}

\title{
Methods
}

Thirty-four healthy Chinese volunteers were recruited for skin biopsy to establish the reference range of intraepidermal nerve fibre density (IENFD). Eighty-nine patients with type 2 diabetes from the Department of Endocrinology at Nanjing Drum Tower Hospital between December 2015 and April 2020 were included in the final study. Metabolic and neurological indexes were evaluated at baseline. Diabetic cardiovascular autonomic function was tested through cardiovascular autonomic reflex tests (CARTs). Seventeen pure SFN subjects and 9 mixed diabetic polyneuropathy (DPN) subjects were reassessed after the follow-up.

\section{Results}

Levels of $\mathrm{HbA}_{1 c}$ and postprandial blood glucose were lower ( $\mathrm{P}=0.005$ and $\mathrm{P}=0.041$, respectively), while postprandial C-peptide and insulin were higher ( $\mathrm{P}=0.001$ and $\mathrm{P}=0.019$, respectively) in the pure SFN group than in the mixed DPN group. Regarding the CARTs, the mixed DPN group obtained the highest score, indicating the worst cardiovascular autonomic neuropathy (CAN). Among the four CART items, postural BP change was lower while deep breathing max-min was higher in the pure SFN group than in the mixed DPN group $(\mathrm{P}=0.023$ and $\mathrm{P}=0.040$, respectively). A partial correlation 
showed that there was a negative correlation between IENFD of the distal leg and CART scores $(r=-0.513, P=0.001)$ after adjusting for age and duration of diabetes. Only vitamin B12 ( $p=0.028)$ and motor nerve conduction velocity (MCV) of the common peroneal nerve $(p=0.045)$ were increased in the 17 patients with pure SFN after the follow-up. However, MCVs of the common peroneal nerve $(p=0.025)$ and tibial nerve $(p=0.047)$ were decreased at the final visit in the mixed DPN group.

\section{Conclusions}

Better islet function and cardiovascular autonomic function were observed in patients with pure SFN compared with mixed DPN. CART scores were negatively correlated with IENFD in the distal leg even after adjusting for age and duration of diabetes. The metabolic and neurological indexes remained relatively stable in the follow-up of pure SFN subjects.

\section{KEYWORDS}

Small fibre neuropathy, diabetic polyneuropathy, cardiovascular autonomic neuropathy, type 2 diabetes mellitus, skin biopsy

\section{Background}

A recent epidemiological survey indicated that approximately 1.56 billion people suffer from diabetes in mainland China[1]. The prevalence of diabetic polyneuropathy (DPN) is approximately $30 \%$ among those with diabetes, and it increases the risk of foot ulcers and amputation[2]. Small fibre neuropathy (SFN) is a type of DPN with a diameter below $7 \mu \mathrm{m}$, and it usually impairs unmyelinated Cfibres and thinly myelinated small Aס-fibres[3]. The main effects associated with SFN are autonomic neuropathy and paraesthesia related to pain, numbness, coldness, and burning sensation, while the nerve conduction velocity (NCV) is usually normal. Skin biopsy is an effective method to diagnose SFN with high specificity and sensitivity. Although skin biopsy is considered the "gold standard" for SFN, it has not been widely used at the Department of Endocrinology in China due to the invasive nature of the procedure. Moreover, we found that SFN was also common in diabetic patients with large nerve fibre neuropathy manifested as abnormal skin biopsy results and NCV. In this study, diabetic patients receiving skin biopsies were enrolled to analyse the metabolic and neurological characteristics, including cardiovascular autonomic function, among different groups. Additionally, we explored correlated factors that may have an impact on intraepidermal nerve fibre density (IENFD) for the early recognition of patients with diabetic neuropathy. In addition, the follow-up of patients with pure SFN and mixed DPN was conducted to understand their prognosis. 


\section{Methods}

\section{Subject recruitment}

A total of 103 diabetic patients complaining of symptoms related to SFN, aged between 20 and 80 years, were enrolled from the Department of Endocrinology at Nanjing Drum Tower Hospital between December 2015 and April 2020. Finally, 89 patients with type 2 diabetes were included in this study. Diabetes was diagnosed according to the WHO criteria[4]. Pure SFN was defined as abnormal IENFD and normal NCV. Mixed DPN was defined as abnormal IENFD and NCV at the same time. Exclusion criteria included patients with type 1 diabetes, peripheral vascular disease, foot ulcer, history of stroke, thyroid disease, vitamin B12 deficiency, and other causes of peripheral neuropathy. (Figure 1)

\section{Neuropathy Symptom Score (NSS) and Numerical Pain Rating Scale (NPRS)}

All the participants received the NSS and the NPRS. For the NSS, patients were asked about symptoms in the lower limbs such as burning, numbness or tingling, when the symptoms usually happened and ways to alleviate the discomfort. The maximum score was 9. A symptom score of 3-4 was defined as mild symptoms; 5-6, moderate symptoms; and 7-9, severe symptoms[5]. An 11-point NPRS was used to evaluate pain intensity in patients with DPN, where $0=$ no pain and $10=$ worst possible pain[6].

\section{Nerve conduction velocity (NCV) test}

The NCV test was performed by a specialized technician at a room temperature of $26^{\circ} \mathrm{C}$, and NCV was considered abnormal if more than two nerve conduction velocities were less than the reference value. A patient with abnormal NCV and a symptom or sign of neuropathy was defined as having confirmed diabetic sensorimotor polyneuropathy (DSPN)[7].

\section{Cardiovascular autonomic reflex tests (CARTs)}

CARTs are used for the assessment of cardiovascular autonomic neuropathy (CAN), including blood pressure variability in response to standing up (postural BP change), heart rate variability (HRV) during deep breathing (HRV_deep breathing), HRV during the Valsalva manoeuvre (Valsalva ratio) and HRV during the lying-to-standing test (30:15 test). For each test, the grade was as follows: normal (score $=0)$, borderline (score $=0.5$ ), or abnormal (score $=1$ ). The patients with a total score $\geq 2$ were defined as patients with CAN. 


\section{SUDOSCAN test}

SUDOSCAN (Impeto Medical, Paris, France) is a non-invasive mobile device to measure autonomic neuropathy in relation to sudomotor function. The patients placed palms and feet on detecting stainless steel electrodes, and a low direct voltage was applied. Quantitative results were measured as hand and feet electrochemical skin conductance levels (HESC and FESC, $\mu S$ ). The severity of sudomotor dysfunction was classified as follows: none (ESC $>60)$, moderate $(40 \leq \mathrm{ESC} \leq 60)$, or severe $(\mathrm{ESC}<40)$.

\section{Skin biopsy}

After local anaesthesia with lidocaine, skin biopsy was performed at the proximal thigh (10 $\mathrm{cm}$ above the lateral malleolus) and distal thigh $(20 \mathrm{~cm}$ above the greater trochanter) by using a 4-mm disposable punch. Then, the incisions were packed with gauze. The skin usually began to scab in two or three days. Aseptic techniques and timely treatment were required during the skin biopsy.

\section{Frozen section preparation}

Skin punch biopsies were dehydrated in $30 \%$ sucrose solution before fixation with $4 \%$ paraformaldehyde for $24 \mathrm{~h}$. Then, biopsy specimens were embedded in OCT compound (SAKURA Tissue-Tek ${ }^{\circledR}$ O.C.T. Compound) and frozen at $-20^{\circ} \mathrm{C}$. Tissue blocks were cut into $50-\mu \mathrm{m}$ slices on a frozen section machine, and each microslide contained 3 discontinuous slices.

\section{Immunofluorescence and epidermal nerve counting}

Microslides covered with skin biopsy slices were left at room temperature (RT) for 30 min and then rinsed in PBS for 10 min three times. PBS was used for rinsing. Slices were permeabilized for $4 \mathrm{~h}$ with $0.3 \%$ Triton-X100 and incubated with a rabbit monoclonal antibody against PGP9.5 (1:1000; Abcam (ab108986) overnight at $4^{\circ} \mathrm{C}$ ). PBST (PBS containing 0.1\% Tween 20) was used for rinsing, followed by Cy3-conjugated goat anti-rabbit antibody (1:2000; Servicebio, $2 \mathrm{~h}$ at RT). Nuclei were stained with DAPI solution for $10 \mathrm{~min}$ at RT in the dark ( $50 \mu \mathrm{l}$ for each sample; Servicebio). An antifade solution was applied to the slices by the coverslip and sealed with clear nail polish.

Images were acquired using laser scanning confocal microscopy (TCS-SP8, Leica, Germany) and overlaid by LAS X software (Leica, Germany). Each slice was scanned 
through the Z-axis every $5 \mu \mathrm{m}$. The quantification of IENFD was performed according to European Federation of Neurological Societies (EFNS) guidelines, and nerves crossing the basement membrane of the epidermis were regarded as effective counts[8]. IENFD=the number of skin nerve fibre counts / the length of the corresponding section $(\mathrm{mm})$.

\section{Statistical analysis}

Data are presented as the mean \pm standard deviation (SD) or mean (range). All analyses were performed using SPSS 22.0 (Chicago, IL). For continuous variables, Student's ttest and one-way ANOVA were used for normally distributed variables, while the Wilcoxon rank-sum test and Kruskal-Wails $\mathrm{H}$ test were used for nonnormally distributed variables. The chi-square test or Fisher's exact test was used for dichotomous variables. ImageJ software 1.50 (National Institutes of Health, Bethesda, Maryland, USA) was used to count the length of the epidermis. P-values $\leq 0.05$ were considered statistically significant.

\section{Results}

\section{Establishment of IENFD reference range}

Thirty-four healthy Chinese volunteers, including 18 men and 16 women, were recruited for this study. The mean age of the subjects was $51.7 \pm 14.5$ years, and the mean $\mathrm{HbA}_{1 \mathrm{c}}$ was $5.4 \pm 0.4 \%$. The mean IENFD at the proximal thigh was $20.7 \pm 6.0 / \mathrm{mm}$ (mean \pm SD; lower 5 th percentile: $10.0 / \mathrm{mm}$ ). The mean IENFD at the distal thigh was $11.3 \pm 3.5 / \mathrm{mm}$ (mean $\pm \mathrm{SD}$; lower 5 th percentile: $8.1 / \mathrm{mm}$ ).

\section{Comparison of normal IENFD between different sexes and ages}

There were no differences in the distal thigh and proximal thigh measures between males and females. Moreover, the subjects were sorted into groups based on their age: 20 50 years and 51 80 years. No significant differences were seen between the two groups (Tables 1 and 2).

\section{Clinical characteristics of subjects participating in the skin biopsy}

Figure 2 shows representative PGP9.5 immunofluorescence in human skin biopsy. IENFD was significantly lower in a diabetic patient with SFN (Figure 2a) than in a 
healthy subject (Figure $2 b$ ).

Patients with type 2 diabetes were classified into the non-DPN, pure SFN and mixed DPN groups according to the established IENFD reference range and NCV test (Table 3). There were no differences in age, sex, body mass index (BMI), or the duration of diabetes among the three groups. The percentages of family history of diabetes, smoking, and alcohol intake were also similar. Blood pressure and resting heart rate were higher in the mixed DPN group than in the non-DPN group (all $P \leq 0.05$ ).

Additionally, the levels of $\mathrm{HbA}_{1 \mathrm{c}}$ and postprandial blood glucose were lower $(\mathrm{P}=0.005$ and $\mathrm{P}=0.041$, respectively), while postprandial $\mathrm{C}$-peptide and insulin were higher $(P=0.001$ and $P=0.019$ for each) in the pure SFN group than in the mixed DPN group. Regarding microvascular complications, the albumin-to-creatinine ratio (ACR) was higher in the mixed DPN group than in the non-DPN group ( $P=0.016)$. The value of $25(\mathrm{OH})$-VitD was lower in the mixed SFN group than in the non-DPN group $(P=0.014)$. No differences were seen in the lipid profiles, or eGFR, folate, or vitamin B12 levels among the three groups (Table 4).

Neuropathy examinations including IENFD, NSS, NPRS, CART scores, SUDOSCAN and NCV tests were performed on the subjects with type 2 diabetes. The IENFD of the proximal and distal leg was significantly lower in the pure SFN group and the mixed DPN group than in the non-DPN group ( $P=0.004$ and $P=0.000$, respectively), but there was no significant difference between the pure SFN group and the mixed DPN group. Motor nerve conduction velocity (MCV) was the lowest in the mixed DPN group, while NSS, NPRS and average hands and feet ESC in SUDOSCAN did not show significant differences among the three groups.

For the CARTs, the mixed DPN group obtained the highest score, indicating the worst CAN. Among the four items of the CARTs, postural BP change was lower, while deep breathing max-min was higher in the pure SFN group than in the mixed DPN group $(P=0.023$ and $P=0.040$, respectively). The percentage of CAN was significantly higher in the mixed DPN group than in the non-DPN group and the pure SFN group $(P=0.003)$ (Table 5).

\section{Follow-up of pure SFN and mixed DPN subjects}

To understand the outcome of pure SFN and mixed DPN subjects, follow-up was conducted after the collected baseline, and the follow-up ended on October $28^{\text {th }}, 2020$. Seventeen pure SFN patients were included with an average age of $55.0 \pm 7.5$ years, diabetes duration of $9.0 \pm 5.7$ years, BMI of $25.0 \pm 2.9 \mathrm{~kg} / \mathrm{m}^{2}$, and a median follow-up of $15(12,20)$ months. Nine mixed DPN patients were included with an average age of $62.9 \pm 11.1$ years, duration of $15.3 \pm 9.5$ years, BMI of $24.4 \pm 3.1 \mathrm{~kg} / \mathrm{m}^{2}$, and a median follow-up of $12(8.5,16.5)$ months. Biochemical and neurological indexes were 
assessed during the follow-up.

For both groups, no changes were seen in glucose and lipid profiles, ACR and eGFR at the final visit. Among micronutrients, only vitamin B12 was increased at the final visit in the pure SFN group ( $p=0.028$ ). Regarding neurological indexes, the NSS, NPRS, CARTs, SUDOSCAN and NCV tests were conducted during the follow-up. The MCVs of the common peroneal nerve $(p=0.025)$ and tibial nerve $(p=0.047)$ were decreased in the mixed DPN group. However, the MCV of the common peroneal nerve showed an upward trend in the pure SFN group ( $p=0.045$ ) (Table 7).

\section{Discussion}

\section{Application of skin biopsy in the diagnosis of diabetic small fibre neuropathy}

In the present study, we reported an overall evaluation of the metabolic indexes in Chinese type 2 diabetic patients with SFN based on skin biopsy. In addition, neurological tests, including cardiovascular autonomic function, were assessed in these subjects.

IENFD is an important parameter for evaluating the severity of SFN by calculating the number of skin nerves per unit length[9]. However, its reference range varies based on race, districts, and staining methods[10]. First, the reference range of IENFD in our district was established by enrolling healthy subjects receiving skin biopsy. The lower 5th percentile of IENFD at the proximal thigh was $10.0 / \mathrm{mm}$, and for the distal thigh, it was $8.1 / \mathrm{mm}$. Kennedy et al[11, 12] reported that the lower 5 th percentile of IENFD at the distal leg was $20.0 / \mathrm{mm}$ in subjects aged 20-59 years, while the number was $11.8 / \mathrm{mm}$ in subjects over 60 years. However, conversely, McArthur et al[13] found no sex or age effect in the IENFD of healthy subjects. There was no significant difference in IENFD between $20 \sim 50$ years and 51 80 years in our study, so we used a unified cutoff value to diagnose SFN.

Although the IENFD in patients in the pure SFN group was significantly lower than that in the non-DPN group, there was no significant difference between the pure SFN group and the mixed DPN group, which indicated that SFN may have a unique pathophysiology rather than simply being an "early stage" of polyneuropathy.

\section{Clinical factors related to pure SFN and mixed DPN}

The pure SFN group manifested better islet function, including lower $\mathrm{HbA}_{1 c}$ and PBG accompanied by higher postprandial insulin and C-peptide compared to the mixed DPN group. However, the eGFR and ACR showed no differences between the two 
groups, indicating that a longer follow-up period was needed to assess diabetic microangiopathy.

We noticed that the level of vitamin D was lower in the mixed DPN group than in the non-DPN group, which was in line with several previous studies[14-16]. Possible mechanisms include vitamin D protecting Schwann cells against advanced glycation end product (AGE)-induced apoptosis[17] and promoting proangiogenic molecules[18]. Further studies are needed to focus on vitamin D in the protection against diabetic neuropathy and the related molecular mechanisms.

\section{Association of CARTs and diabetic small fibre neuropathy}

Several studies have paid more attention to diabetic cardiovascular autonomic neuropathy (DCAN) in recent years. As an important type of autonomic neuropathy, DCAN can increase the mortality associated with cardiovascular diseases[19-21]. However, it usually has an insidious onset and is easily neglected by clinicians. CARTs are the gold standard for screening for DCAN[22]. Heart rate variations, including deep breathing, the Valsalva manoeuvre, and lying-to-standing, are used to test parasympathetic function. Orthostatic hypotension indicates impairment in sympathetic function[23].

In this study, the item of deep breathing max-min, a marker of the excitability of the parasympathetic nervous system (PNS), was lower in the pure SFN group than in the non-DPN group, although their total CART scores showed no difference. Additionally, partial correlation showed a negative correlation between IENFD of the distal leg and CART scores even after adjusting for age and duration of diabetes. In general, CARTs are vital to early screening for DCAN in diabetic patients considering long-term severe complications.

\section{Follow-up and outcome of pure SFN and mixed DPN subjects}

After the baseline study of SFN confirmed by skin biopsy, we conducted a follow-up of patients with pure SFN and mixed DPN. No changes were seen in islet function or lipid and kidney profiles between the two groups. Vitamin B12 and MCV of the common peroneal nerve were increased at the final visit in the pure SFN group. Conversely, the MCVs of the common peroneal nerve and tibial nerve were decreased in the mixed DPN group.

In a previous study, MacDonald S et al[24] reported a retrospective analysis of 101 patients with biopsy-confirmed SFN, and the average follow-up was 6.2 years. They found that small fibre neuropathy tended to be stable and rarely affected ambulation and employment status. However, electrophysiological tests were not assessed in 
MacDonald's study, and the aetiology of SFN was diverse, including hyperglycaemia, immune dysfunction, vitamin B12 deficiency, and so on. Løseth S et al[25] evaluated the progression of 35 type 1 and 24 type 2 diabetic neuropathy patients, and there was minimal progression of large fibre neuropathy in both groups.

In our study, the MCV of common peroneal nerve increased in the pure SFN group. The reason for the improvement of large fibre neuropathy in pure SFN patients is still unknown, and it may partly due to the individual pathophysiological mechanism of SFN. Although $\mathrm{HbA1C}$ and fasting C-peptide ameliorated in the mixed DPN group, the MCV of lower limbs deteriorated at the final visit. The phenomenon was also consistent with the previous studies related to intensive blood glucose control and neurological complications, which meant tighter blood glucose control did not bring to lighter neuropathy [26-28]. Therefore, more attention should be paid to the early recognition of SFN in diabetic patients with a high risk of large fibre neuropathy.

Regarding vitamin B12, the differences between the two groups may be attributed to the usage of nutritional supplements in some patients. Additionally, we noticed that age at the initial visit was higher in the mixed DPN group, which meant that the large fibre nerve was probably vulnerable because of an age-related factor. Thus, a longer follow-up time is needed in future studies related to SFN.

\section{Limitations and strengths of this study}

The study has several limitations that should be addressed. First, the number of healthy subjects was relatively small, and due to heterogeneity in IENFD, the diagnosis of small fibre neuropathy could only be defined by the reference range determined from the local district. Second, the subjects were recruited from the inpatient department, which may not represent the overall population of patients with small fibre neuropathy. Third, the follow-up was relatively short to assess the features of patients with pure SFN and mixed DPN.

In summary, the strength of the study was the initial and thorough assessment of SFN based on skin biopsy in a relatively large population of Chinese diabetic patients. Additionally, we found a negative correlation between CART scores and IENFD in the distal leg even after adjusting for the age and duration of diabetes. Finally, metabolic and neurological indexes remained stable in the pure SFN subjects with type 2 diabetes during the follow-up.

\section{Conclusion}

In this study, we reported the clinical characteristics of Chinese diabetic patients with 
small fibre neuropathy based on skin biopsy. Better islet function and cardiovascular autonomic function were observed in those with pure SFN compared with mixed DPN. CART scores were negatively correlated with IENFD in the distal leg even after adjusting for age and duration of diabetes. It seems that the metabolic and neurological indexes were relatively stable in the follow-up of pure SFN subjects. Additionally, it is necessary to attach importance to early screening of CAN in diabetic patients.

\section{Additional files}

\section{Abbreviations}

ACR: albumin-to-creatinine ratio; AGEs: advanced glycation end products; BMI: body mass index; BP: blood pressure; CAN: cardiovascular autonomic neuropathy; CARTs: cardiovascular autonomic reflex tests; CRP: C-reactive protein; DBP: diastolic blood pressure; eGFR: estimated glomerular filtration rate; FESC: feet electrochemical skin conductance; FPG: fasting plasma glucose; HDL-C: high-density lipoprotein cholesterol; HESC: hands electrochemical skin conductance; HOMA-IR: homeostasis model assessment of insulin resistance; MCV: motor nerve conduction velocity; NCV: nerve conduction velocity; NPRS: Numerical Pain Rating Scale; HRV: heart rate variability; NSS: Neuropathy Symptom Score; IENFD: intraepidermal nerve fibre density; LDL-C: low-density lipoprotein cholesterol; PBG: 2-hour postprandial blood glucose; PCP: 2-hour postprandial Cpeptide; PINS: 2-hour postprandial insulin; RHR: resting heart rate; SBP: systolic blood pressure; TC: total cholesterol; TG: triglycerides.

\section{Authors' contributions}

D.Z and Y.B. conceived and designed the study. C.L., W.N, C.C., and J.J. conducted the skin biopsy. C.L. performed all data analysis and drafted the manuscript. W.C. performed the NCV test and W.W. contributed to the revision of the manuscript. All the other authors critically reviewed and approved the final manuscript.

\section{Author details}

1 Department of Endocrinology, Drum Tower Hospital Affiliated to Nanjing University Medical School, Nanjing, China. 2 Department of Osteoporosis, Lianyungang First People's Hospital, Lianyungang, China. 3 Department of Endocrinology, The First Affiliated Hospital, Sun Yat-sen University, Guangzhou, China. 4 Department of Endocrinology, Fujian Provincial Hospital, Fuzhou, Fujian, China. 


\section{Acknowledgements}

Special thanks to Hongyu Huang, Quan Zhang, and Lu Zhou for photographing stained slices and Dr. Lan Wang for the theoretical guidance in neurology. Donghui Yang participated in patientrelated instrumental examinations.

\section{Competing interests}

The authors declare that they have no competing interests.

\section{Availability of data and materials}

The datasets used and/or analysed in the current study are available from the corresponding authors upon reasonable request.

\section{Consent for publication}

Not applicable.

\section{Ethics approval and consent to participate}

The study (NCT04071535) was approved by the Ethics Committee of Drum Tower Hospital Affiliated to Nanjing University Medical School and was performed according to the Declaration of Helsinki.

\section{Funding}

This work was supported by National Natural Science Foundation of China Grant Awards [81570737, 81570736] and the Key Research and Development Program of Jiangsu Province of China [BE2015604].

\section{References}

1. Li Y, Teng D, Shi X, Qin G, Qin Y, Quan H, Shi B, Sun H, Ba J, Chen B et al: Prevalence of diabetes recorded in mainland China using 2018 diagnostic criteria from the American Diabetes Association: national cross sectional study. BMJ (Clinical research ed) $2020,369: \mathrm{m} 997$.

2. Callaghan BC, Cheng HT, Stables CL, Smith AL, Feldman EL: Diabetic neuropathy: clinical manifestations and current treatments. Lancet Neurology 2012, 11(6):521-534. 
3. Andreas, Themistocleous, Juan, Ramirez, Jordi, Serra, David: The clinical approach to small fibre neuropathy and painful channelopathy. Practical Neurology 2014.

4. Alberti K, Zimmet P: Definition, diagnosis and classification of diabetes mellitus and its complications: report of a WHO consultation. Diabetic Medicine 1999, 15(7):539553.

5. Young MJ, Boulton AJ, MacLeod AF, Williams DR, Sonksen PH: A multicentre study of the prevalence of diabetic peripheral neuropathy in the United Kingdom hospital clinic population. Diabetologia 1993, 36(2):150-154.

6. Farrar JT, Young JP, Jr., LaMoreaux L, Werth JL, Poole RM: Clinical importance of changes in chronic pain intensity measured on an 11-point numerical pain rating scale. Pain 2001, 94(2):149-158.

7. Tesfaye S, Boulton AJDyck PJ, Freeman R, Horowitz M, Kempler P, Lauria G, Malik RA, Spallone V, Vinik A, Bernardi L: Diabetic neuropathies: update on definitions, diagnostic criteria, estimation of severity, and treatments. J Diabetes Care 2010, 33(10):2285-2293.

8. Hsieh ST: EFNS guidelines on the use of skin biopsy in the diagnosis of peripheral neuropathy. European journal of neurology 2007, 13(12):e9.

9. Lauria G, Cornblath DR, Johansson O, McArthur JC, Mellgren SI, Nolano M, Rosenberg N, Sommer C: EFNS guidelines on the use of skin biopsy in the diagnosis of peripheral neuropathy. European journal of neurology 2005, 12(10):747-758.

10. Shikuma CM, McArthur JC, Ebenezer GJ, Ananworanich J, Teeratakulpisarn N, Jadwattanakul T, Valcour VG, Bennett K, Phanuphak N: Ethnic differences in epidermal nerve fiber density. Muscle \& nerve 2013, 48(3):462-464.

11. Kennedy WR, Wendelschafer-Crabb G, Johnson T: Quantitation of epidermal nerves in diabetic neuropathy. Neurology 1996, 47(4):1042-1048.

12. Periquet MI, Novak V, Collins MP, Nagaraja HN, Erdem S, Nash SM, Freimer ML, Sahenk Z, Kissel JT, Mendell JR: Painful sensory neuropathy: prospective evaluation using skin biopsy. Neurology 1999, 53(8):1641-1647.

13. McArthur JC, Stocks EA, Hauer P, Cornblath DR, Griffin JW: Epidermal nerve fiber density: normative reference range and diagnostic efficiency. Archives of neurology 1998, 55(12):1513-1520.

14. Almurdhi MM, Brown SJ, Bowling FL, Boulton AJM, Jeziorska M, Malik RA, Reeves ND: Altered walking strategy and increased unsteadiness in participants with impaired glucose tolerance and Type 2 diabetes relates to small-fibre neuropathy but not vitamin D deficiency. Diabetic medicine : a journal of the British Diabetic Association 2017, 34(6):839-845. 
15. Niu Y, Li J, Peng R, Zhao X, Wu J, Tang Q: Low vitamin D is associated with diabetes peripheral neuropathy in older but not in young and middle-aged patients. Diabetes/metabolism research and reviews 2019, 35(6):e3162.

16. Alam U, Petropoulos IN, Ponirakis G, Ferdousi M, Asghar O, Jeziorska M, Marshall A, Boulton AJ, Efron N, Malik RA: Vitamin D Deficiency is Associated With Painful Diabetic Neuropathy. Diabetes/metabolism research and reviews 2020:e3361.

17. Xu S, Li J, Zhai M, Yao X, Liu H, Deng T, Cai H, Zhang W, Zhang W, Lou J et al: 1,25$(\mathrm{OH})(2) \mathrm{D}(3)$ protects Schwann cells against advanced glycation end productsinduced apoptosis through PKA-NF-KB pathway. Life sciences 2019, 225:107-116.

18. Trujillo V, Marín-Luevano P, González-Curiel I, Rodríguez-Carlos A, Ramírez-Reyes M, Layseca-Espinosa E, Enciso-Moreno JA, Díaz L, Rivas-Santiago B: Calcitriol promotes proangiogenic molecules in keratinocytes in a diabetic foot ulcer model. The Journal of steroid biochemistry and molecular biology 2017, 174:303-311.

19. Kuehl M, Stevens MJ: Cardiovascular autonomic neuropathies as complications of diabetes mellitus. Nature reviews Endocrinology 2012, 8(7):405-416.

20. Yun JS, Park YM, Cha SA, Ahn YB, Ko SH: Progression of cardiovascular autonomic neuropathy and cardiovascular disease in type 2 diabetes. Cardiovasc Diabetol 2018, 17(1):109.

21. Jun JE, Lee SE, Choi MS, Park SW, Hwang YC, Kim JH: Clinical factors associated with the recovery of cardiovascular autonomic neuropathy in patients with type 2 diabetes mellitus. Cardiovasc Diabetol 2019, 18(1):29.

22. Ewing DJ, Campbell IW, Clarke BF: Assessment of cardiovascular effects in diabetic autonomic neuropathy and prognostic implications. Annals of internal medicine 1980, 92(2 Pt 2):308-311.

23. Spallone V, Ziegler D, Freeman R, Bernardi L, Frontoni S, Pop-Busui R, Stevens M, Kempler P, Hilsted J, Tesfaye S et al: Cardiovascular autonomic neuropathy in diabetes: clinical impact, assessment, diagnosis, and management. Diabetes/metabolism research and reviews 2011, 27(7):639-653.

24. MacDonald S, Sharma TL, Li J, Polston D, Li Y: Longitudinal follow-up of biopsy-proven small fiber neuropathy. Muscle \& nerve 2019, 60(4):376-381.

25. Løseth S, Stålberg EV, Lindal S, Olsen E, Jorde R, Mellgren SI: Small and large fiber neuropathy in those with type 1 and type 2 diabetes: a 5-year follow-up study. J Peripher Nerv Syst 2016, 21(1):15-21.

26. Group AC, Patel A, MacMahon S, Chalmers J, Neal B, Billot L, Woodward M, Marre M, Cooper M, Glasziou P et al: Intensive blood glucose control and vascular outcomes in patients with type 2 diabetes. N Engl J Med 2008, 358(24):2560-2572. 
27. Duckworth W, Abraira C, Moritz T, Reda D, Emanuele N, Reaven PD, Zieve FJ, Marks J, Davis SN, Hayward R et al: Glucose control and vascular complications in veterans with type 2 diabetes. N Engl J Med 2009, 360(2):129-139.

28. Ismail-Beigi F, Craven T, Banerji MA, Basile J, Calles J, Cohen RM, Cuddihy R, Cushman WC, Genuth S, Grimm RH, Jr. et al: Effect of intensive treatment of hyperglycaemia on microvascular outcomes in type 2 diabetes: an analysis of the ACCORD randomised trial. Lancet 2010, 376(9739):419-430. 
Figures

Skin biopsy, $n=103$

Excluded, $n=9$

6 Latent autoimmune

diabetes in adults

1 Hypercortisolism

1 Primary aldosteronism

1 Hepatitis B chronic carrier

T2DM, $n=94$
Excluded, $\mathrm{n}=5$

5 Lacked nerve conduction velocity test

Final, $\mathrm{n}=89$

7 Non-DPN

37 Pure SFN

45 Mixed DPN

Figure 1

Flowchart for participating subject inclusion 


\section{Figure 2}

PGP9.5 Immunofluorescence in human skin biopsy (a: Healthy subject. b: Diabetic patient diagnosed with small fibre neuropathy. Blue area: DAPI staining indicating cell nucleus. Red lines: PGP9.5-labelled small nerve fibre.) 